\title{
Guest editorial to the Theme Section on enterprise modelling
}

\author{
Tony Clark - Florian Matthes • Balbir Barn • \\ Alan Brown
}

Published online: 23 March 2013

(C) Springer-Verlag Berlin Heidelberg 2013

\section{Introduction}

Modern organizations rely on complex configurations of distributed IT systems that implement key business processes, provide databases, data warehousing, and business intelligence. The current business environment requires organizations to comply with a range of externally defined regulations such as Sarbanes-Oxley and BASEL II. Organizations need to be increasingly agile, robust, and be able to react to complex events, possibly in terms of dynamic reconfiguration.

In order to satisfy these complex requirements, large organizations are increasingly using enterprise modelling (EM) technologies to analyse their business units, processes, resources and IT systems, and to show how these elements satisfy the goals of the business. EM describes all aspects of the construction and analysis of organizational models and supports enterprise use cases including:

Business alignment where elements of a business are shown to meet its goals and in particular establishing that the IT systems and processes that run the business are consistent with the goals set by the Chief Executive. Business change management where as-is and to-be models are used to plan how a business is to be changed based on a precise definition of business component dependencies.

\section{T. Clark $(\varangle) \cdot$ B. Barn}

Middlesex University, London, UK

e-mail: t.n.clark@mdx.ac.uk

F. Matthes

TU München, Munich, Germany

e-mail: matthes@in.tum.de

A. Brown

IBM Software Group, Raleigh, USA

e-mail: awbrown@us.ibm.com
Governance and compliance where models are used to show that processes and information structures are in place to comply with regulations.

Acquisitions and mergers where models are used to analyse the effect of combining two or more businesses. Outsourcing where models can be used to establish the equivalence between a particular slice of an organization and a service provider.

Enterprise resource planning in which models are used to analyse the use of resources within a business and to show that given quality criteria are achieved.

Quality analysis where features such as quality of service (QoS), organizational resilience and failure rates can be measured in terms of their impact in a business.

\section{Selected papers for this Theme Section}

A call for papers was announced in 2011 and attracted 29 initial abstracts. All submissions were reviewed by at least 3 experts in the field and were subjected to a rigorous selection process, in many cases involving a second round of review in the light of suggestions from the reviewers. The final selection of 10 articles reflects the level of interest in this area of modelling and the high quality of both the submissions and the feedback from the reviewers.

Organizational processes need to be understood by a large number of people and from many different perspectives and levels of abstraction from the CEO through to the software maintenance team. If modelling is to help in this regard, it must be possible to support multiple stakeholder viewpoints for the same process and to keep the viewpoints in sync when the process is modified. The article A Case Study on Consistency Management of Business and IT Process Models in Banking by Branco, Xiong, Czarnecki, Kster and 
Voelzer, uses a real-world consistency management case study to provide an outline of the research challenges in this area.

The article Multi-Perspective Enterprise Modeling: Foundational Concepts, Prospects and Future Research Challenges by Frank, also addresses the issue of multiple stakeholder perspectives in enterprise models and described a method (MEMO) that is supported by a meta-modelling tool. The approach advocates the use of domain-specific languages to help to support the construction and presentation of the underlying models in ways that are meaningful to different stakeholders.

Multiple stakeholders have expertise that requires different levels of abstraction with respect to different domains. Systems engineering has supported multiple problems and solution domains through the development of technologies and approaches based on Domain Specific Languages (DSL): languages whose concepts and semantics are tailored to support a restricted domain rather than general purpose applications. Given the size of enterprises and the number of stakeholder perspectives, there is clearly a need for DSL approaches. The article Extending Enterprise Architecture Modeling Languages for Domain Specificity and Collaboration. Application to Telecommunications Service Design by Chiprianov, Kermarrec, Rouvrais and Simonin, described how DSL techniques can be applied to Enterprise Architecture within the telecomms domain.

Business processes represent one of the most wide-spread uses of modelling within organizations. Given the rate of change within an organization, it is important that the construction and maintenance of business process models are efficient and of high quality. The article Literature review of reuse in business process modeling by Zaaboub Haddar, Makni and Ben Abdallah provides an overview of the key features that can be reused, the criteria for choosing the reusable artefacts and outlines future directions for research in this area.

Enterprise architecture (EA) uses a framework for representing and analysing the what, why and how of an organization. TOGAF is a leading EA framework and ArchiMate is a leading technology that supports EA modelling. The article A Reference Requirements Set for Public Service Provision Enterprise Architectures by Tambouris, Kaliva, Liaros and Tarabanis, uses TOGAF and ArchiMate to address electronic government (eGov) and to produce a set of reference requirements for eGov.

An important distinction between systems modelling and enterprise modelling is that the goals or intentions of an enterprise are usually considered to be an intrinsic feature and should be modelled together with information, structure and process models. Once we have modelled the goals of an organization, we can use business intelligence (BI) to determine whether our systems are aligned to the strategic direction of the enterprise. The article Strategic Business Modeling: Representation and Reasoning by Horkoff, Barone, Jiang, $\mathrm{Yu}$, Amyot, Borgida and Mylopoulos, proposed a modelling language called BIM for modelling and reasoning about BI.

Enterprise architecture models must represent much more than the what and the how that is traditionally expressed in system models. Technologies for EA have been emerging over recent years and a leading EA language and associated toolset is ArchiMate. Although ArchiMate provides many of the key modelling elements required to express EA features, the article Bridging Value Modelling to ArchiMate via Transaction Modelling by Kinderen, Gaaloul and Proper, proposes an extension that allows models to represent the value of transactions in business processes.

A key feature of modern organizations is that they change on a regular basis because of some need to increase the value of the business, create a new product, or to respond to some change in the business environment. An important EA usecase is the ability to represent an enterprise $a s$-is and to-be and to be able to analyse the difference in order to validate the change. The article From Enterprise Architecture to Business Models and back by Iacob, Meertens, Jonkers, Quartel, Nieuwenhuis and van Sinderen, describes how ArchiMate is related to business process models and the resulting technology can be analysed in terms of the costs and benefits of an as-is and to-be migration.

Modern organizations are huge and involve many different characteristics that must be taken into account when performing as-is analysis, for example response time, availability, usage, accuracy, etc. Although modelling approaches exist for the different characteristics, they tend to be point solutions and often do not integrate. A model-based technique that addresses this issue is to integrate at the language level via meta-models. The article An enterprise architecture framework for multi-attribute information systems analysis by Nrman, Buschle and Ekstedt, shows how the meta-models for ArchiMate can be extended with meta-models that support four EA properties and subsequently analysed through the integrated constraint language p-OCL.

It is rare that enterprise modelling techniques are applied to design a new organization; they are most often used to understand the motivation, structure and behaviour of an existing enterprise. Given an existing organization, an enterprise architect faces the problem of how to analyse the existing structures and systems in order to create a valid model that can be used for subsequent analysis. The article Assessing Event Correlation in Non-Process-Aware Information Systems by Perez-Castillo, Weber, Garca-Rodrguez, Piattini and Pinggera, describes a process whereby the events recorded in the logs of IT systems can be used to extract business process models. 
We wish to thank all of the reviewers for their excellent timely feedback and all the authors of the articles for their high-quality submissions and for responding positively to the reviewers' comments.

\section{Research challenges}

Modelling has a great deal to offer in addressing the challenges faced by the modern enterprise. As demonstrated by the articles in this Theme Section, there is a great deal of diverse modelling research in this area. Given the breadth and depth of the challenges involved, there are a vast number of research opportunities in applying modelling techniques to problems of understanding, analysing, modifying, operationalizing and maintaining enterprises, including:

Meta-models Language engineering, including domainspecific languages (DSLs) and language integration, will play a large part in EM due to the diverse number of stakeholders involved. DSLs must allow each stakeholder to engage with the models without requiring technological expertise.

Tools EM tools are currently in their infancy. In addition to facilitating multiple stakeholders, the tools must integrate with a wide range of IT systems.

Analytics EM technologies must support a wide range of diverse analysis use-cases including quality-based analytics, business intelligence and dependency analysis. An important challenge is to present the results of multiple types of analysis in ways in which stakeholders can engage.

Transformations Model transformation is a fairly mature technology that can be used to support EM migration and the generation of code and IT system configuration data. Transformations are traditionally performed in ways that target a single IT system, however, EM presents a much wider challenge involving multiple-integrated systems. Integration Model-based interoperability is a challenge that is currently supported by open standards such as UML. In order to address the challenges of EM, much greater levels of integration will be required that links models of the business goals to detailed models of the underlying IT systems.

The idea of using models as tools to design, analyse, transform and ultimately run a business is gaining significant momentum in practice and academia. A major motivation is to help stakeholders to master the ever-increasing complexity of organizations by suitable abstractions from multiple disciplines. This Theme Section represents a step in this direction.

\section{Author Biographies}

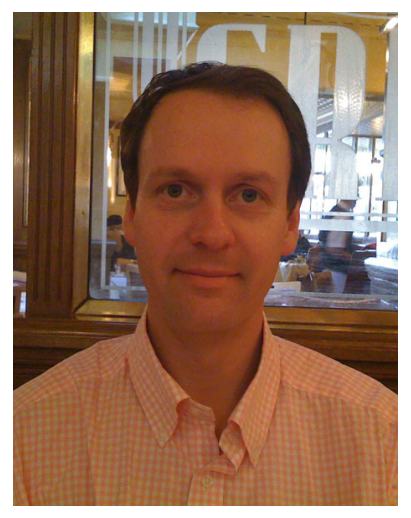

Tony Clark is Professor of Informatics and Head of the Computer Science Department in the School of Science and Technology at Middlesex University. Tony has experience of working in both Academia and Industry on a range of software projects and consultancies. While at Marconi Research Ltd (1985-1994) he worked in both Software Engineering and Knowledge Based Systems, and was responsible for designing novel systems for recognising aircraft behaviour and fusing sonar data, and for designing and implementing an AI Toolkit, the first of its kind in the UK. From 1994 to 2003, Tony was an academic first at Bradford University and then at King's College London. During this time, he worked on languages for objectoriented specification and design including contributing to a range of Industry standards (UML 2.0, MDA, QVT, MOF) through participation in the Object Management Group (OMG). During this time, Tony's group at King's College London developed tooling for object-oriented language development. This tooling work together with the development of a novel approach to meta-modelling and language design on the UML 2.0 standard led to a spin-out company which Tony co-founded in 2003 and served as Technical Director 2003-2008. Tony was co-chair of MODELS 2011, has been an editor for several special journal issues including IEEE Software and SoSyM. His current work addresses the development and analysis of models for enterprise architecture. Further information can be found at http://www.eis.mdx.ac.uk/staffpages/ tonyclark/.

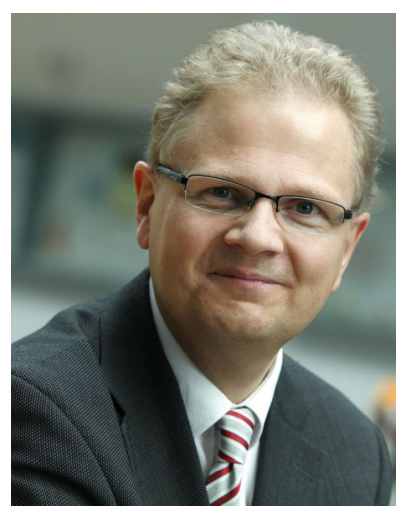

Florian Matthes holds the chair Software Engineering for Business Information Systems at the Faculty of Informatics of Technische Universität München since 2002. The current focus of his research is on enterprise architecture management, social software engineering and interactive information visulizations. Earlier stations of his academic career are the Digital Systems Research Center (now HP SRC Classic) in Palo Alto, USA, and the Technical University Hamburg-Harburg. He is member of the editorial board of the MIT Press Cooperative Information Systems series, member of the advisory board of the EDBT Foundation, head of the software architecture working group of the Gesellschaft für Informatik, member of the advisory board of the Ernst Denert-Foundation for Software Engineering. He served as (co-)chair of several international workshops and conferences in the area of enterprise architecture, enterprise transformation and software engineering. In all these roles, he puts special emphasis on the cooperation between practitioners and scientists in informatics and information systems. Parallel to his academic career, he is cofounder and chairman of several spin-off companies with more than 180 employees and scientific advisor of the centre of innovation and business creation at TU München. Further information can be found at http://wwwmatthes.in.tum.de. 


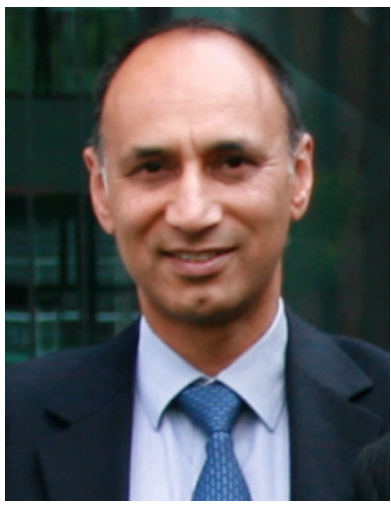

Balbir Barn is Professor of Software Engineering and Deputy Dean in the School of Science and Technology at Middlesex University, UK. Balbir has over 15 years commercial research experience working in research labs at Texas Instruments and Sterling Software where he was involved in the research and design of leading software products such as the IEFTM. His research is focused on model driven software engineering where the goal is to use models as abstractions and execution environments to support, for example, enterprise architecture and application integration using complex events. He has led numerous externally funded projects which apply model-driven principles to business processes, learning theories and more recently the theory building aspects to model-driven engineering.

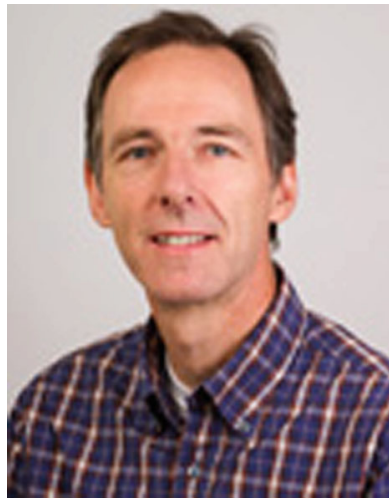

Alan Brown is Professor of Entrepreneurship and Innovation in the Surrey Business School where he leads activities in the area of corporate entrepreneurship and open innovation models. In addition to teaching activities, he focuses on innovation in a number of practical research areas with regard to global enterprise software delivery, agile software supply chains, and the investigation of 'open commercial' software delivery models. Alan is also an IBM
Distinguished Engineer at IBM Rational software where he consults on business strategy, agile software delivery, and new business models. Alans previous assignment was IBM Rational CTO for Europe. In the past 20 years working in both industry and academia in the USA, UK, and Spain, Alan has extensive experience in service-based design, component-based development, software engineering environments, and enterprise application development tools. He has published over 60 papers, authored five books, and edited an additional three books. Alan holds a PhD in Computing Science from the University of Newcastle-upon-Tyne, UK. 